\title{
Análise da tipologia facial através do Índice Vert de Ricketts e do Índice Morfológico Facial
} Analysis of facial typology using Ricketts Vert Index and the Facial Index Análisis de biotipo facial segun el índice Vert de Ricketts y el Ângulo de Apertura Facial

\section{Maria Carolina Bandeira MACENA ${ }^{1}$}

Fátima Roneiva Alves FONSECA ${ }^{1}$

Pedro Douglas Souto HENRIQUES ${ }^{2}$ Myllena Alves XAVIER ${ }^{3}$

Caio Pimenteira UCHÔA ${ }^{4}$

Anna Katarina Costa LEITE $^{4}$

Alexandre Durval LEMOS 5

${ }^{1}$ Doutora e Mestre em Ortodontia - Universidade Federal de Campina Grande (UFCG) Patos - PB, Brasil

${ }^{2}$ Graduado em Odontologia - Universidade Federal de Campina Grande (UFCG) Patos - PB, Brasil

${ }^{3}$ Mestre em Ciência e Tecnologia em Saúde - Universidade Estadual da Paraíba (UEPB) Campina Grande - PB, Brasil

${ }^{4}$ Discente do Curso de Odontologia - Universidade Federal de Campina Grande (UFCG) Patos - PB, Brasil

${ }^{5}$ Doutor em Ortodontia e Mestre em Fisiologia Oral - Universidade Estadual da Paraíba (UEPB) - Campina Grande - PB, Brasil

\section{Resumo}

Este estudo teve como objetivo avaliar a concordância no diagnóstico da tipologia facial de pacientes em tratamento ortodôntico, através de medidas cefalométricas laterais na Análise de Ricketts e do Îndice Morfológico Facial. A amostra foi constituída por 60 indivíduos de ambos os sexos, acima de 18 anos, pertencentes ao curso de pós-graduação em ortodontia das FIP (Faculdades Integradas de Patos - PB, Brasil), avaliados por meio da antropometria direta (Índice Morfológico Facial) e da antropometria indireta (Análise do Índice Vert de Ricketts). Os resultados foram analisados estatisticamente por meio do coeficiente Kappa ponderado. Obteve-se uma pobre concordância entre os dois métodos na determinação do tipo facial. Concluiu-se que a antropometria direta, método consolidado como importante recurso na determinação do tipo facial a partir da altura e largura faciais, não encontrou concordância com os valores do índice Vert da Análise de Ricketts.

Descritores: Índices; Ortodontia; Antropometria.

\section{Abstract}

This study aimed to evaluate the agreement in the diagnosis of the facial typology of patients undergoing orthodontic treatment, through lateral cephalometric measurements in the Ricketts Vert Index and the Facial Index. The sample consisted of 60 individuals of both sexes, over 18 years old, belonging to the postgraduate course in orthodontics of the FIP (Integrated Faculties of Patos - PB, Brazil), evaluated by means of direct anthropometry (Facial Morphological Index) and indirect anthropometry (Ricketts Vert Index). The results were statistically analyzed using the weighted Kappa coefficient. Poor agreement was obtained between the two methods in determining facial type. It was concluded that direct anthropometry, a method consolidated as an important resource in determining facial type from facial height and width, did not find agreement with the Ricketts Vert Index values.

Descriptors: Indexes; Orthodontics; Anthropometry.

\section{Resumen}

Este estudio tuvo como objetivo evaluar la concordancia en el diagnóstico de la tipología facial de pacientes sometidos a tratamiento de ortodoncia, mediante medidas cefalométricas laterales en el Índice Vert de Ricketts y el ângulo de Apertura Facial. La muestra estuvo conformada por 60 individuos de ambos sexos, mayores de 18 años, pertenecientes al posgrado en Ortodoncia de la FIP (Facultades Integradas de Patos - SP, Brasil), evaluados mediante antropometría directa (Índice Morfológico Facial) y antropometría indirecta (Índice Vert de Ricketts). Los resultados se analizaron estadísticamente mediante el coeficiente Kappa ponderado. Se obtuvo una escasa concordancia entre los dos métodos para determinar el tipo de rostro. Se concluyó que la antropometría directa, un método consolidado como un recurso importante en la determinación del tipo facial a partir de la altura y el ancho del rostro, no encontró concordancia con los valores del Índice Vert de Ricketts.

Descriptores: Índices; Ortodoncia; Antropometría.

INTRODUÇÃO

O diagnóstico do padrão craniofacial do paciente é imprescindível na Ortodontia, pois influencia na decisão do plano de tratamento e no prognóstico em cada caso. Os diferentes padrões faciais devem ser determinados com precisão, visto que o padrão esquelético reflete no processo dentoalveolar ${ }^{1}$ e a análise imprópria destes pode causar alterações faciais que interferem na estética e na estabilidade dos resultados ${ }^{2}$.

Durante a consulta odontológica além do exame clínico dentário, o ortodontista faz uma análise facial preliminar que ocorre de forma direta e permite observar a adaptação dos tecidos moles ao perfil esquelético do paciente3, com auxílio de radiografias e fotografias interpretadas através de medidas angulares e lineares é feita uma análise indireta para classificar os perfis faciais estéticos ${ }^{2-4}$.

A análise de Ricketts é utilizada para identificar padrões de crescimento através do índice de Vert, por meio de cinco grandezas cefalométricas (altura facial anteroinferior, arco mandibular, eixo facial, plano mandibular e profundidade facial). Os resultados provenientes dessas medidas classificam a face em seis tipos (mesofacial, dolicofacial, dolicofacial leve, dolicofacial grave, braquifacial, braquifacial leve e braquifacial grave). O indivíduo braquifacial possui a face curta e alargada com arcos dentários arredondados, o dolicofacial tem a face mais longa e estreita com arcos dentais relativamente atrésicos, o mesofacial apresenta características entres os dois tipos ${ }^{5}$.

O Índice Vert classifica o valor 0 (zero) como sendo do tipo mesofacial, valores negativos como dolicofacial (-0,5 dólico suave e a partir de -2 dólico severo) e valores positivos como braquifacial $(0,5$ braquifacial e a partir de 1 braquifacial severo $)^{6}$.O Índice Morfológico 
Facial (IMF), propôs a classificação dos tipos faciais em Hipereuriprosopo e Euriprosopo (crescimento facial no sentido horizontal), Mesoprosopos (crescimento facial equilibrado) e Leptoprosopo e Hiperleptoprosopo (crescimento facial no sentido vertical), baseado na avaliação frontal do paciente ${ }^{7}$.

A antropometria é uma técnica de mensuração vertical da face angular e linear, baseado em pontos pré-definidos que permitem obter importantes dados semiológicos para caracterização do crescimento facial. As medições podem ser feitas diretamente no paciente, esta técnica é propícia por apresentar baixo risco, ser simples, confiável e acessível ${ }^{8}$.

O objetivo deste estudo foi avaliar a concordância no diagnóstico da tipologia facial de pacientes em tratamento ortodôntico, através do traçado cefalométrico lateral de Ricketts (Índice Vert) e da análise facial frontal, por meio do IMF (Índice Morfológico Facial).

\section{MATERIAL E MÉTODO}

Esta pesquisa foi aprovada pelo comitê de ética das FIP sobre o protocolo CAAE: 49199815.1.0000.5181. A amostra foi composta por 60 pacientes acima de 18 anos, de ambos os sexos, em tratamento nas clínicas de Especialização em Ortodontia da Faculdade Integrada de Patos (FIP) e que possuíssem na sua documentação a análise de Ricketts. A idade de 18 anos foi selecionada por indicar o final de grandes modificações craniofaciais devidas ao processo de crescimento e desenvolvimento.

As documentações foram adquiridas de um mesmo serviço de radiologia para minimizar erros de padronização na posição natural da cabeça durante as tomadas radiográficas da cefalometria lateral. Pacientes com histórico de tratamentos ortodônticos anteriores ou de cirurgias ortognáticas foram excluídos; não se levou em consideração o tipo de oclusão dentária.

O índice Vert de Ricketts de cada telerradiografia foi computado através da Análise lateral de Ricketts solicitada do serviço de documentação selecionado. A partir da análise cefalométrica de Ricketts, os sujeitos foram classificados com relação à tipologia de facial em dolicofacial (índice VERT menor que 0,5 ), mesofacial (valores entre -0,5 e +0,5) e braquifacial (VERT maior que 0,5).

A análise antropométrica frontal foi realizada pelo próprio pesquisador, previamente calibrado, através do registro das medidas faciais bi zigomática (zi-zi) e Násio-Gnátio (nagn) dos pacientes sentados em posição confortável e cabeça mantida em posição natural; os dentes em oclusão cêntrica ${ }^{7}$. As medições foram realizadas através do paquímetro digital King Tools com capacidade de $150 \mathrm{~mm}$, resolução $0,01 \mathrm{~mm}$, código: 502.150BL (Figura 1). Anteriormente a coleta de dados foi efetuada uma modificação na face de medição, substituindo as pontas ativas do paquímetro digital, implantando duas espátulas de madeira, com as extremidades alongadas, a fim de realizar os procedimentos de medição ${ }^{7-9}$. Por fim foi calculado o Índice Morfológico Facial na-gn/zi-zi ${ }^{10}$.

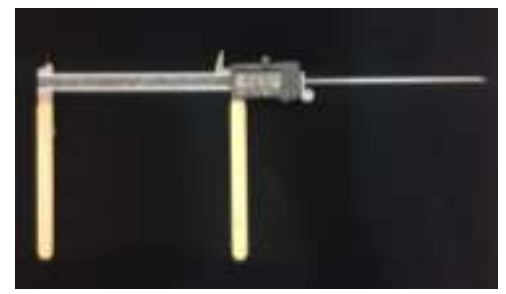

Figura 1: Paquímetro digital adaptado com espátulas de madeira.

Previamente à coleta de dados, realizouse um estudo piloto e os procedimentos de calibração. Dez pacientes foram avaliados e 30 dias após reavaliados pelo examinador usando o Índice Morfológico Facial para estabelecer o tipo facial. A concordância intraexaminador foi analisada mediante o teste Kappa, obtendo valor no intervalo de $0,80-1,00$, o que indica haver uma concordância excelente.

- Análise dos resultados

Realizou-se a análise estatística descritiva objetivando caracterizar a amostra. Em seguida, os achados relativos a tipologia facial obtidos por ambos os métodos foram submetidos à análise estatística por meio do coeficiente Kappa ponderado para avaliação da concordância entre os métodos de acordo com o sexo dos participantes.

Os valores do coeficiente Kappa foram interpretados como 14: concordância pobre $(\mathrm{k}<0)$, ligeira concordância $(\mathrm{k}=0-0,20)$, concordância fraca $(k=0,21-0,40)$, concordância moderada $\quad(k=0,41-0,60), \quad$ concordância substancial $(k=0,60-0,80)$ e concordância excelente $(k>0,80)$. Todas as análises foram conduzidas usando o software SPSS Statistics versão 20.0 .

RESULTADOS

A Tabela 1 exibe a distribuição dos participantes de acordo com o sexo e o perfil facial. A maioria era do sexo feminino $(n=37$; $61,7 \%$ ). De acordo com o índice VERT, 36 $(60,0 \%)$ indivíduos foram classificados como braquifaciais, ao passo que o IMF revelou que $56(93,3 \%)$ eram dolicofaciais.

A Tabela 2 apresenta a análise comparativa dos resultados obtidos por meio do IMF e do Índice Vert de Ricketts de acordo com 
o perfil facial, demostrando que não houve qualquer concordância entre os conceitos de tipologia braquifacial ou mesofacial entre os métodos; e que apenas em 9 casos (15\%) apresentaram concordância entre os métodos para o padrão dolicofacial. Verificou-se uma concordância pobre $(\mathrm{k}<0)$.

Tabela 1. Distribuição dos participantes de acordo com o sexo e o perfil facial.

\begin{tabular}{|c|c|c|}
\hline Variáveis & $\mathbf{n}$ & $\%$ \\
\hline \multicolumn{3}{|l|}{ Sexo } \\
\hline Feminino & 37 & 61,7 \\
\hline Masculino & 23 & 38,3 \\
\hline \multicolumn{3}{|l|}{ Perfil Facial (Índice Vert) } \\
\hline Braquifacial & 36 & 60,0 \\
\hline Mesofacial & 15 & 25,0 \\
\hline Dólicofacial & 9 & 15,0 \\
\hline \multicolumn{3}{|l|}{ Perfil Facial (IMF) } \\
\hline Braquifacial & o & 0,0 \\
\hline Mesofacial & 4 & 6,7 \\
\hline Dolicofacial & 56 & 93,3 \\
\hline TOTAL & 60 & 100 \\
\hline
\end{tabular}

Tabela 2. Análise comparativa dos resultados obtidos por meio do IMF e do Índice Vert de Ricketts de acordo com o perfil facial.

\begin{tabular}{|c|c|c|c|c|}
\hline \multicolumn{5}{|c|}{ Perfil facial (Índice Vert de Ricketts) } \\
\hline Perfil facial & Braquifacial & Mesofacial & Dolicofacial & Total \\
\hline (IMF) & n (\%) & $\mathrm{n}(\%)$ & n (\%) & $\mathrm{n}(\%)$ \\
\hline Mesofacial & $4(100,0)$ & $\mathrm{o}(0,0)$ & $\mathrm{o}(0,0)$ & $4(100,0)$ \\
\hline Dolicofacial & $32(57,1)$ & $15(26,8)$ & $9(16,1)$ & $56(100,0)$ \\
\hline TOTAL & $36(60,0)$ & $15(25,0)$ & $9(15,0)$ & $60(100,0)$ \\
\hline
\end{tabular}

A Tabela 3 mostra a análise comparativa dos resultados obtidos por meio do IMF e do Índice Vert de Ricketts de acordo com o perfil facial para o sexo feminino. Verificou-se uma concordância pobre $(k<0)$. De acordo com a classificação da face obtida a partir do Índice Vert de Ricketts, o tipo facial mais frequente no sexo feminino foi o braquifacial $(n=21)$. Em contraste, o IMF revelou que o tipo facial mais frequente foi o dolicofacial $(n=34)$.

A Tabela 4 mostra a análise comparativa dos resultados obtidos por meio do IMF e do Índice Vert de Ricketts de acordo com o perfil facial para o sexo masculino. Verificou-se uma ligeira concordância $(k=0-0,20)$. De acordo com a classificação da face obtida a partir do Índice Vert de Ricketts, o tipo facial mais frequente no sexo masculino foi o braquifacial ( $\mathrm{n}$ = 15). Em contraste, o IMF revelou que o tipo facial mais frequente foi o dolicofacial $(n=22)$.

Tabela 3. Análise comparativa dos resultados obtidos por meio do IMF e do Índice Vert de Ricketts de acordo com o perfil facial para o sexo feminino.

\begin{tabular}{|c|c|c|c|c|}
\hline \multicolumn{5}{|c|}{ Perfil facial (Índice Vert de Ricketts) } \\
\hline \multirow{2}{*}{$\begin{array}{l}\text { Perfil facial } \\
\text { (IMF) }\end{array}$} & Braquifacial & Mesofacial & Dolicofacial & Total \\
\hline & $\mathrm{n}(\%)$ & $\mathrm{n}(\%)$ & n (\%) & $\mathrm{n}(\%)$ \\
\hline Mesofacial & $3(100,0)$ & $\mathrm{o}(0,0)$ & $0(0,0)$ & $3(100,0)$ \\
\hline Dolicofacial & $18(52,9)$ & $11(32,4)$ & $5(14,7)$ & $34(100,0)$ \\
\hline TOTAL & $21(56,8)$ & $11(29,7)$ & $5(13,5)$ & $37(100,0)$ \\
\hline
\end{tabular}

Tabela 4. Análise comparativa dos resultados obtidos por meio do IMF e do Índice Vert de Ricketts de acordo com o perfil facial para o sexo masculino.

\begin{tabular}{c|l|l|l|l}
\hline \multicolumn{5}{c}{ Perfil facial (Índice Vert de Ricketts) } \\
\hline \multirow{2}{*}{$\begin{array}{l}\text { Perfil facial } \\
(\text { IMF) }\end{array}$} & Braquifacial & Mesofacial & Dolicofacial & Total \\
\cline { 2 - 5 } & $\mathrm{n}(\%)$ & $\mathrm{n}(\%)$ & $\mathrm{n}(\%)$ & $\mathrm{n}(\%)$ \\
\hline Mesofacial & $1(100,0)$ & $\mathrm{O}(0,0)$ & $\mathrm{o}(0,0)$ & $1(100,0)$ \\
\hline Dolicofacial & $14(63,6)$ & $4(18,2)$ & $4(18,2)$ & $22(100,0)$ \\
\hline TOTAL & $15(65,2)$ & $4(17,4)$ & $4(17,4)$ & $23(100,0)$ \\
\hline Nota. Kappa $=0,000$. & & &
\end{tabular}

DISCUSSÃO

Existem diversos parâmetros

esqueléticos para avaliar o padrão de crescimento vertical que por muitas vezes apontam resultados conflitantes e dificultam a determinação de um diagnóstico específico. Considerando que o crescimento vertical da face é o último a terminar, analisar a discrepância facial na dimensão vertical é importante no diagnóstico, planejamento e, sobretudo na prevenção de recidiva após correção da má oclusão ${ }^{11}$.

Semelhante a outros estudos, foi encontrado um número maior de pessoas do sexo feminino na amostra analisada. Esse resultado pode estar relacionado ao fato das mulheres terem maior preocupação com a estética e a saúde $\mathrm{e}^{7-12}$.

$\mathrm{Na}$ presente pesquisa o padrão facial mais prevalente foi o braquifacial em ambos os sexos, esse dado corrobora com outros autores $^{13,14}$. Golalipour et al. ${ }^{15}$, afirmam que as diferentes regiões geográficas e as mudanças raciais são relevantes na determinação dos padrões faciais e apontam que indivíduos da América do sul com proximidade ao Oceano Pacífico tende a ser braquicefálicos. Nota-se também uma menor prevalência de padrões dolicofaciais na amostra, semelhante os achados de Berwig et al. ${ }^{16}$, que objetivou avaliar as medidas antropométricas orofaciais segundo o tipo facial de crianças.

Nascimento et al. ${ }^{17}$ afirmam que as medidas antropométricas orofaciais são influenciadas quanto ao gênero, sendo que homens apresentam maiores valores quando comparados a mulheres. Esse dado atestou-se em nossos resultados onde $(65,2 \%)$ da amostra masculina é braquifacial frente a $(56,8 \%)$ da amostra feminina.

Com relação à concordância entre o Índice Vert e o Índice Morfológico Facial os resultados obtidos revelaram uma limitada concordância conforme tem sido evidenciado na literatura ${ }^{18}$. Ao avaliar sexo feminino a concordância apresentou-se ainda mais irrelevante quando comparada aos resultados obtidos para o sexo masculino. Diferente de outro estudo que mostrou não haver concordância entre os dois métodos para o sexo masculino e um nível de concordância significante entre os dois métodos da determinação do tipo facial para 0 sexo feminino ${ }^{7}$.

A interpretação cefalométrica é limitada na ortodontia, pois a telerradiografia fornece a imagem das estruturas craniofaciais em formato 
bidimensional (2D) $)^{19}$. Ao considerar que o Índice Vert é calculado sobre a telerradiografia apenas de um lado e o IMF é mensurado diretamente na face do paciente em posição frontal pode-se inferir uma possível explicação em relação à insatisfatória concordância dos índices estudados na presente pesquisa ${ }^{18}$. As diferentes posições da cabeça na realização das avaliações também interferem, visto que as medidas angulares utilizadas para o cálculo do Índice Vert e a análise facial são alteradas de acordo com o posicionamento ${ }^{20}$.

Outra justificativa para a restrita concordância apresentada nesse estudo seria a utilização do ÍMF para a determinação do tipo facial sem considerar os diferentes tipos de etnias e ascendências. Dado que a morfologia craniofacial é considerada multifatorial, e o desenvolvimento facial é influenciado por um número de genes e por vários fatores ambientais ${ }^{21}$.

\section{CONCLUSÃO}

Os resultados deste estudo mostraram uma pobre concordância na determinação dos tipos faciais obtidos através no Índice Morfológico Facial com os obtidos por meio do índice VERT de Ricketts, na cefalometria lateral. Portanto, a antropometria direta, embora esteja consolidada como importante recurso na determinação do tipo facial a partir da altura e largura faciais, não substitui a análise cefalométrica de Ricketts.

\section{REFERÊNCIAS}

1. Queiroz GV, Rino J, Paiva JB, Capelozza L. Analysis of reliability, accuracy, sensitivity and predictive value of a subjective method to classify facial pattern in adults. Dental Press $\mathrm{J}$ Orthod. 2016;21(6):58-66.

2. Benedicto EN, Kairalla SA, Kaieda AK, Miranda SL, Torres FC, Paranhos LR. Determinação do padrão esquelético vertical da face. Rev Bras Cir Craniomaxilofac. 2011;14(1):44-9.

3. Rocha TL, Pinzan A, Bronfman CN, Henriques JFC, Janson G. Estudo cefalométrico comparativo da análise de Ricketts para jovens brasileiros leucodermas, melanodermas e mestiços (feodermas) com oclusão normal. Ortodontia. 2015; 48(2):168-78.

4. Quintão APA, Vitral RWF. Estudo comparativo entre cefalometria manual e computadorizada (análise de Steiner, Tweed e Downs) em telerradiografias laterais. $\mathrm{HU}$ Rev.2010;36(2):95-9.

5. Claro CAA, Abrao JR, Braga SA. Association between overbite and craniofacial growth pattern. Braz oral res. 2010;24(4):425-32.

6. Ricketts, Robert M and Rocky Mountain Data Systems Orthodontic diagnosis and planning: their roles in preventive and rehabilitative dentistry. Rocky Mountain/Orthodontics, Denver, Colo; 1982.

7. Ramires RR, Ferreira LP, Marchesan IQ, Cattoni DM, Silva MAA. Medidas faciais antropométricas de adultos segundo tipo facial e sexo. Rev CEFAC. 2011;13(2):245-52.

8. Furtado IA, Agostinho HR. Apresentação do Método Anátomo-Clínico de Faciometria Directa em Crianças com Objectivos Propedêuticos. Acta Med Port. 2014;27(4): 458-66.

9. Sies ML, Farias SR, Vieira MM. Respiração oral: relação entre o tipo facial e a oclusão dentária em adolescentes. Rev Soc Bras Fonoaudiol, 2007;12(3):191-98.

10. Rakosi T, Jonas I, Graber TM. Exames clìnicos especiais. In: Ortodontia e ortopedia facial: diagnÛstico. Porto Alegre: Artmed; 1999.

11. Ahmed M, Shaikh A, Fida M. Diagnostic performance of various cephalometric parameters for the assessment of vertical growth pattern. Dental Press J. Orthod. 2016; 21(4):41-9.

12. Perez I, Chavez AK, Ponce D. Applicability of the Ricketts' posteroanterior cephalometry for sex determination using logistic regression analysis in Hispano American Peruvians. J Forensic Dent Sci. 2016;8(2):111-25.

13. Bavia PF, Vilanova LSR, Garcia RCMR. Craniofacial Morphology Affects Bite Force in Patients with Painful Temporomandibular Disorders. Braz Dent J.2016;27(5):619-24.

14. Rosa APB, Rodrigues EG, Torres FC, Comar LP, Alonso MBCC, Triviño T. Correlação entre as formas dos arcos dentários e os tipos faciais. Arch Health Invest. 2016;5 (Spec Issue 6):10.

15. Golalipour MJ, Haidari K, Jahanshahli M, Frahani, MR. The shapes of head and face in normal male newborns in South- east of Caspian Sea(Iran- Gorgan). J Anat Soc. 2003; 52(28):31-43.

16. Berwig LC, Figueiredo FSP, Bolzan GP, Milanesi JM, Marquezan M, Busanello-Stella AR et al. Medidas antropométricas orofaciais de crianças segundo o tipo facial. Rev CEFAC. 2017;19(1):63-8.

17. Nascimento WV, Cassiani RA, Dantas RO. Efeito do gênero, da altura corporal e da etnia nas medidas antropométricas orofaciais. CoDAS. 2013;25(2):149-53.

18. Bolzan GP, Berwing LC, Prade LS, Weinmann ARM, Moraes AB, Silva AMT. Concordância entre o método antropométrico e cefalométrico na classificação do tipo facial. Rev CEFAC. 2014;16(1):222-27.

19. Roque-Torres G, Meneses-López A, NorbertoBóscolo F, De Almeida SM, Haiter-Neto F. La tomografía computarizada cone beam en la ortodoncia, ortopedia facial y funcional Rev Estomatol Herediana. 2015;25(1):61-78. 
20. Haiter-Neto F, Oliveira SS, Casanova MS, Caldas MP. Telerradiografias obtidas em posição natural da cabeça alteram as grandezas cefalométricas? Rev Dent Press Ortodon Ortoped Facial. 2007;12(4):117-23.

21. Bedoya A, Osorio JC, Tamayo J.A. Biotipo morfológico facial en tres grupos étnicos colombianos: una nueva clasificación por medio del índice facial. Int J Morphol. 2012;30(2): 677-82.

\section{CONFLITO DE INTERESSES}

Os autores declaram não haver conflitos de interesse

\section{AUTOR PARA CORRESPONDÊNCIA}

Myllena Alves Xavier

Universidade Estadual da Paraíba (UEPB)

Rua Baraúnas, 351 - Universitário,

58429-000 Campina Grande - PB, Brasil

Tel (83)998309588

Email:myllenalves@hotmail.com 